\title{
Quantum Information Paradox: Real or Fictitious?
}

\author{
Abhas Mitra* \\ Theoretical Astrophysics Section, Bhabha Atomic Research Centre, Mumbai, India
}

(Dated: November 3, 2018)

\begin{abstract}
One of the outstanding puzzles of theoretical physics is whether quantum information indeed gets lost in the case of Black Hole (BH) evaporation or accretion. Let us recall that Quantum Mechanics (QM) demands an upper limit on the acceleration of a test particle. On the other hand, it is pointed out here that, if a Schwarzschild BH would exist, the acceleration of the test particle would blow up at the event horizon in violation of $Q M$. Thus the concept of an exact $\mathrm{BH}$ is in contradiction of $\mathrm{QM}$ and quantum gravity (QG). It is also reminded that the mass of a BH actually appears as an INTEGRATION CONSTANT of Einstein equations. And it has been shown that the value of this integration constant is actually zero! Thus even classically, there cannot be finite mass BHs though zero mass $\mathrm{BH}$ is allowed. It has been further shown that during continued gravitational collapse, radiation emanating from the contracting object gets trapped within it by the runaway gravitational field. As a consequence, the contracting body attains a quasi-static state where outward trapped radiation pressure gets balanced by inward gravitational pull and the ideal classical $\mathrm{BH}$ state is never formed in a finite proper time. In other words, continued gravitational collapse results in an "Eternally Collapsing Object" which is a ball of hot plasma and which is asymptotically approaching the true $\mathrm{BH}$ state with $M=0$ after radiating away its entire mass energy. And if we include QM, this contraction must halt at a radius suggested by highest QM acceleration. In any case no EH is ever formed and in reality, there is no quantum information paradox.
\end{abstract}

PACS numbers: 03.67.-a, 04.20.Dw, 04.40.-b

Keywords: Quantum Information Paradox - Black Hole - Eternally Collapsing Object

\section{INTRODUCTION: UPPER LIMIT ON ACCELERATION IN QUANTUM GRAVITY}

Quantum Gravity imposes a fundamental unit of proper length called Planck Length:

$$
l_{p}=\sqrt{\frac{\hbar G}{c^{3}}}
$$

and a fundamental unit of time called Planck Time $\tau_{p}=l_{p} / c$. Accordingly any QG should impose a Maximal Acceleration:

$$
a_{p}=\frac{\text { Maximum Speed }}{\text { Minimum Proper Time }} \sim \frac{c^{2}}{l_{P}}=\frac{c^{7 / 2}}{\sqrt{\hbar G}}
$$

Detail considerations yields 1,2 ,

$$
a_{p}=\frac{c^{2}}{2 l_{P}}=\frac{c^{7 / 2}}{2 \sqrt{\hbar G}}
$$

Thus $Q M$ does not allow occurrence of infinite proper acceleration. Some preliminary formulation of QG indeed mentions of Maximal Acceleration[3].

\section{Proper Acceleration Due to Gravity}

The Invariant Proper Acceleration on a test particle around lying on the surface of a spherical body of mass $M$ and Radius $R_{0}[\underline{4}]$

$$
a=\sqrt{-a^{i} a_{i}}=\frac{G M}{R_{0}^{2} \sqrt{1-2 G M / R_{0} c^{2}}}
$$


And if the body is instead a Schwarzschild black hole with $R_{0}=2 G M / c^{2}$, one would have $a=\infty[4]$ !! Note that since $a$ is an invariant/scalar, its value is independent of coordinates and even a free falling test particle would experience infinite acceleration. Clearly, such a situation would be in violation of the inherent concept of a natural QG upper limit of acceleration $a_{p}$. Thus no meaningfully formulated QG should admit existence of BHs. Hence in properly formulated QG theories, there should be neither any horizon, nor any BH, nor any Hawking Radiation. Thus as per QG, either there must be some physical process to stop continued gravitational collapse at a certain $R_{0}=R_{p}$ where $a \leq a_{p}$ or else, in the case of Schwarzschild BHs, the mass of the BH should be zero: $M=0$. This is so because of two reasons:

- The proper time of formation of a zero mass BH is infinite: $\tau_{\text {formation }} \propto M^{-1 / 2}=\infty$.

- Even if mathematically, one would assume the existence of such a zero mass BH, the proper time of infall of a test particle for reaching the Event Horizon (EH) $\tau_{\text {infall }} \propto M^{-1 / 2}=\infty$.

In this way, the conflict of avoiding an infinite acceleration would be avoided.

\section{Hint From String Theories}

There are some solutions in String Theories which do not admit any horizon or singularity. For instance

- "If one applies the transformation (2.13) to time transformation in Schwarzschild, one obtains a solution in which the horizon becomes a singularity." [5]

Note, horizon itself becomes the singularity only when mass of the $\mathrm{BH}: M=0$ because radius of the $\mathrm{BH}, R_{g}=2 M$ $(G=c=1)$.

- "D1-D5 solutions have neither any horizon nor any singularity." [5]

- "Straight Strings have no Event Horizon" [5].

Some solutions of the string theories specifically suggest that point particle are massless:

- "The consistency of our picture requires that there is one and only one supermultiplet which becomes massless at the singularity" $[\underline{6}]$

"At first sight it may seem surprising that classical black holes can be massless. However this phenomenon has an appealing explanation from 10-D perspective. The IIA(IIB) theory has extremal black twobrane (threebrane) solutions whose mass is proportaional to their area. After Calabi- Yau compactification these may wrap around minimal two (theree) surface and appear as a 4-D black hole. As the area of the surfaces around which they wrap goes to zero, the corresponding $\mathrm{BH}$ becomes massless...." [6].

When $\mathrm{BH}$ is massless, its horizon area $A=0$, and this a unique state with entropy

$$
S=\ln 1=0
$$

Physically a massless BH with zero horizon area confines no information. There is no question of evaporation or information loss in such a case. Nor does a test particle ever reach the horizon of a massless BH $\tau_{\text {infall }} \propto M^{-1 / 2}=\infty$.

In fact, there are specific indications that QG does not actually allow any Hawking Radiation (even if the existence of a $\mathrm{BH}$ would be assumed:

"A New argument is presented confirming the point of view that a Schwarzschild BH formed during a gravitational collapse process does NOT radiate" [7].

- 'Hawking effect may be only a mathematical artifact because it demands singularity of the wave function at event horizon in violation of QM.." [8].

\section{DOES GENERAL RELATIVITY ALLOW FINITE MASS BHS?}

The existence of the vacuum Schwarzschild solution apparently suggests existence of BHs of arbitrary mass. But what is forgotten here is that (i) The "mass" of the $\mathrm{BH}$ appears through an integration constant: $\alpha=2 G M / c^{2}$ or $M=c^{2} \alpha / 2 G$.

(ii) Although symbolically "integration constants" may look to assume arbitrary value, in reality, some of them may have a definite or precise value.

\section{Fixing the value of this Integration Constant}

One may consider the exterior Schwarzschild BH solution in two different coordinates: 
- The original Schwarzschild Solution :

$$
d s^{2}=(1-\alpha / R) d T^{2}-(1-\alpha / R)^{-1} d R^{2}-R^{2}\left(d \theta^{2}+\sin ^{2} \theta d \phi^{2}\right)
$$

- The Eddigton -Finkelstein Solution [9, 10, 11, 12]:

$$
d s^{2}=(1-\alpha / R) d T_{*}^{2} \pm(2 \alpha / R) d T_{*} d R-(1+\alpha / R) d R^{2}-R^{2}\left(d \theta^{2}+\sin ^{2} \theta d \phi^{2}\right)
$$

where

$$
d T_{*}=d T+\frac{\alpha}{R-\alpha} d R
$$

and apply the condition that proper 4-volume remains invariant for any coordinate transformation, i.e.,

$$
\sqrt{-g_{*}} d R d T_{*} d \phi d \theta=\sqrt{-g} d R d T d \theta d \phi
$$

where $g=\operatorname{det}\left|g_{i k}\right|$ and happens to be same in both the cases: $g=g_{*}=-R^{4} \sin ^{2} \theta$. Then the foregoing Eq. yoields $d T_{*}=d T$ which, by virtue of Eq.(98 demands

$$
\alpha=\frac{2 G M}{c^{2}}=0
$$

or $M=0$. This means that though the mass of a Schwarzschild BH which appears to be arbitrary, actually, it is unique: $M \equiv 0$ ! It is important to note that if instead of a "point particle" we would be considering a finite static spherical body of gravitational mass $M$, the above derivation would be invalid even though by Birchoff's theorem, the exterior spacetime would still be described by metric (2). This happens because the coordinate transformation( 8 ) is obtained by integrating the vacuum null geodesic all the way from $R=0$. And this is allowed only when the spacetime is indeed due to a point mass and not due to a finite body. For a finite body, the interior metric would be different from what is indicated by Eqs.(6) and (7). As explained above, $M=0 \mathrm{BHs}$ are never formed, even if they would be assummed to be there, there is no Hawking Radiation!

More importantly, this means that the observed BH Candidates having finite massess cannot be exact BHs though they may resemble Dark/Black like theoretical BHs. This would be so if the Gravitational Redshft of the real BH Candidates, $z \gg 1$. WHY? This is so because observed luminosity of an object falls off as

$$
L_{\text {observed }} \sim(1+z)^{-2} \rightarrow 0 \text { as } z \gg 1
$$

where one defines

$$
z=\left(1-2 G M / R c^{2}\right)^{-1 / 2}-1
$$

Recall here that for ideal BHs, $z=\infty$ and consequently $L_{\text {observed }}=0$.

\section{CONSISTENCY WITH GRAVITATIONAL COLLAPSE}

The metric of a spherically evolving fluid is given by

$$
d s^{2}=g_{00} d t^{2}+g_{r r} d r^{2}-R^{2}\left(d \theta^{2}+\sin ^{2} \theta d \phi^{2}\right)
$$

where $R=R(r, t)$ is the Invariant Circumference coordinate (also called as areal coordinate) and happens to be a scalar. Further, for radial motion with $d \theta=d \phi=0$, the metric becomes

$$
d s^{2}=g_{00} d t^{2}\left(1-x^{2}\right)
$$

where

$$
x=\frac{\sqrt{-g_{r r}} d r}{\sqrt{g_{00}} d t}
$$

We may recast Eq.(14) as

$$
\left(1-x^{2}\right)=\frac{1}{g_{00}} \frac{d s^{2}}{d t^{2}}
$$


The comoving observer at $r=r$ is free to do measurements of not only the fluid element at $r=r$ but also of other objects: If the comoving observer is compared with a static floating boat in a flowing river, the boat can monitor the motion of other boats or the pebbles fixed on the river bed. Here the fixed markers on the river bed are like the background $R=$ fixed markers against which the river flows. Alternatively, the comoving observer may be seen as the driver of a car (local fluid) while the fixed milestones on the roadside constitute the $R=f i x e d=$ scalar grid naturally definable for any spherically symmetric problem. If we intend to find the parameter $x$ for such a $R=$ constant milestone, we will have, $d R=0$, or

$$
d R(r, t)=0=\dot{R} d t+R^{\prime} d r
$$

where an overdot denotes a partial derivative w.r.t. $t$ and a prime denotes a partial derivative w.r.t. $r$. Therefore for the $R=$ constant marker, we find that

$$
\frac{d r}{d t}=-\frac{\dot{R}}{R^{\prime}}
$$

[28] and the corresponding $x=x_{c}$ is

$$
x=x_{c}=\frac{\sqrt{-g_{r r}} d r}{\sqrt{g_{00}} d t}=-\frac{\sqrt{-g_{r r}} \dot{R}}{\sqrt{g_{00}} R^{\prime}}
$$

Using Eq.(16), we also have

$$
\left(1-x_{c}^{2}\right)=\frac{1}{g_{00}} \frac{d s^{2}}{d t^{2}}
$$

Now let us define [13, 14, 15] two auxiliary parameters

$$
\Gamma=\frac{R^{\prime}}{\sqrt{-g_{r r}}} ; \quad U=\frac{\dot{R}}{\sqrt{g_{00}}}
$$

so that the combined Eqs. (18), (19) and (21) yield

$$
x_{c}=-\frac{U}{\Gamma} ; \quad U=-x_{c} \Gamma
$$

As is well known, the gravitational mass of the collapsing (or expanding) fluid is defined through the equation[4, 13, 14,15

$$
\Gamma^{2}=1+U^{2}-\frac{2 M(r, t)}{R}
$$

Then the two foregoing equations can be combined and transposed to obtain

$$
\Gamma^{2}\left(1-x_{c}^{2}\right)=1-\frac{2 M(r, t)}{R}
$$

By using Eqs.(20) and (22) in the foregoing Eq., we obtain

$$
\frac{R^{\prime 2}}{-g_{r r} g_{00}} \frac{d s^{2}}{d t^{2}}=1-\frac{2 M(r, t)}{R}
$$

Recall that the determinant of the metric tensor is always negative [4, 13, 14, 15]:

$$
g=R^{4} \sin ^{2} \theta g_{00} g_{r r} \leq 0
$$

so that we must always have

$$
-g_{r r} g_{00} \geq 0
$$

As mentioned before, all worldlines of material particles or observers must be timelike because the comoving metric (or for that matter even the appropriate Schwarzschild metric) has no supposed "coordinate singularity" unlike the 
vacuum Schwarzschild case. For the signature chosen here, this means, $d s^{2}>0$ for all observers. Then by noting Eq.(27), it follows that the LHS of Eq.(25) is always positive. So must then be the RHS of the same Eq. and which implies that

$$
\frac{2 M(r, t)}{R}<1
$$

This shows in the utmost general fashion that trapped surfaces are not formed in spherical collapse or expansion 4 , 13, 14, 15, 16]. Thus the crucial assumption behind Singularity Theorems of Hawking, Penrose, Geroch, i.e., "Formation of a Trapped Surface" is actually not realized in General Relativity. Therefore, if a fluid would indeed collapse to a singularity at $R \rightarrow 0$, one must have $M \rightarrow 0$ in order that the inequality is honoured. And this is consistent with the result that for BHs, $\alpha=2 G M / R c^{2}=0$ and BHs have unique mass $M \equiv 0$ !

\section{ETERNALLY COLLAPSING OBJECTS}

Two natural questions here would be: 1 . What about the upper limit of White Dwarf and Neutron Star Masses?

Answer: These limits are intended for objects supported by cold quantum degeneracy pressure. On the other hand there is NO UPPER LIMIT for HOT objects supported by RADIATION PRESSURE[17, 18, 19, 20].

2. If there cannot be any finite mass $\mathrm{BH}$ what are the true nature of the observed BH Candidates?

Answer: It has been showed that during continued gravitational collapse, the radiation (photons/neutrinos) emanating from the collapsing object would get almost trapped by the runaway gravitational field. Finally a state would be reached when [17, 18, 19, 20, 21]:

$$
\text { Inward pull of gravity }=\text { Outward trapped radiation pressure }
$$

and consequently, the collapsing object becomes a hot ball of quasistatic plasma where extreme radiation pressure balances the gravity. However, it is still a quasi-static stage and is contracting at infinitesimally slow rate to attain the exact $\mathrm{BH}$ state with $M=0$. It has also been shown that this contraction process continues indefinitely and hence the BH Candidates are Eternally Collapsing Objects (ECOs) rather than true BHs!

\section{OBSERVATIONAL PREDICTION AND VERIFICATION}

As an astrophysical plasma contracts, the frozen in magnetic field increases as $B \propto R^{-2}$ and this is the basic reason that young neutron stars have $B \sim 10^{12-13} \mathrm{G}$. Further in the extreme relativistic stages with $z \gg 1$, local value of $B$ may additionally increase as $B \propto(1+z)$. On this basis, the present author had predicted that the observed BH Candidates, i.e., ECOs will have strong intrinsic magnetic field [4, 13, 20]. In contrast ture chargeless BHs have no intrinsic magnetic field. Consequently, some of the properties as well as accretion geometries around ECOs could resemble, in some way to those of magnetized Neutron Stars rather than (true) BHs. And this prediction has been verified both for stellar mass (galactic) and extragalactic BH Candidates (i.e., ECOs) [22, 23, 24, 25, 26]. In particular, for the extragalactic cases, microlensing observations spanning 20 years have given almost direct proof that, the some of the Quasars, in particular Q0957 + 561, harbour Magnetized ECOS (MECOS) rather than true BHs[25, 26].

\section{CONCLUSION}

Though BH solution is a correct an exact solution, the integration constant defining the mass of a Schwarzschild $\mathrm{BH}$ has the unique value $M=0$. This means that the exact $\mathrm{BH}$ solution represents only an ideal state which can be attained asymptotically during continued gravitational collapse. At any finite proper time, the collapsing object is never, an ideal, exact BH. On the other hand, in the context of classical General Relativity, the actual BH Candidates are likely to be (magnetized) ECOs, a ball of relativistic plasma where inward pull of gravity is balanced by outward radiation pressure. Since all meaningful Quantum Gravity theories must reduce to GR in the low energy regime, the apparent BH like solutions too must be pointing only ideal limiting/asymptotic states which can never exactly occur in Nature. Since there is no exact BH, no Event Horizon, there is no Hawking Radiation and Quantum Information Paradox. As mentioned in the beginning, the existence of an upper limit of a finite proper acceleration $a_{p}$ directly contradicts the notion of exact BHs. 
In an important paper, it was shown that if BHs would be assumed to be present, Quantum Information Paradox would be even more severe than usually considered 27]. However, rather than noting that, the most physical solution to this severe problem would lie in the realization that there cannot be finite mass BHs or Hawking Radiation, the authors suggested far fetched possibilites. But it is hoped that it would be realized that Quantum Information paradox is actually non-existent.

* Electronic address: amitra@barc.gov.in

[1] Arun Pati, Euro Phys Lett., 18 (1992), 285.

[2] Arun Pati, IL Nuovo Cimento B, 107 (1992), 895.

[3] E.R. Caianiello et al., Int. J. Mod. Phys. D, 3(2) (1994), 485.

[4] A. Mitra, Found. Phys. Lett., 15(5) (2002), 439.

[5] G. Horowitz, (1992) (hep-th/9210119)

[6] A. Strominger, Nucl. Phys. B., 451 (1995), 109, (hep-th/954090).

[7] V.A. Belinski, Phys. Lett. A 354 (2006), 249, gr-qc/060713.

[8] M. Rabinowitz, (2004); astro-ph/0412101.

[9] A. Mitra, Adv. Sp. Res., 38(12) (2006), 2917, (astro-ph/0510162).

[10] A. Mitra, J. Math. Phys. 50 (4), (2009), arXiv:0904.4754

[11] A. Mitra (2005), physics/0504076.

[12] A. Mitra, Proc. 11th Marcel Gross Conf. on General Relativity (2006, Berlin, World Scientific)

[13] A. Mitra, Found. Phys. Lett., 13(6) (2000), 543.

[14] A. Mitra, (2004), astro-ph/0408323

[15] A. Mitra, (2005) gr-qc/0512006.

[16] D. Leiter \& S.R. Robertson, Found. Phys. Lett., 16 (2003), 143.

[17] A. Mitra, Mon. Not. R. Astron. Soc. Lett., 367 (2006), L66; gr-qc/061025.

[18] A. Mitra, Mon. Not. R. Astron. Soc., 369 (2006), 492; gr-qc/0603055.

[19] A. Mitra, Phys. Rev. D 74(2) (2006), 02010; gr-qc/0606066.

[20] A. Mitra, New Astronomy, 12(2) (2006), 146; astro-ph/0608178

[21] A. Mitra \& N.K. Glendenning, eScholarship Repository, Lawrence Berkeley National Laboratory, University of California (2006), see, http://repositories.cdlib.org/lbnl/LBNL-59320.

[22] S. Robertson and D. Leiter, Astrophys. J., 565 (2002), 447.

[23] S. Robertson and D. Leiter, Astrophys. J., 569 (2003), L203.

[24] S. Robertson and D. Leiter, Mon. Not. R. Astron. Soc., 350 (2004), 1391.

[25] R. Schild, D. Leiter \& S. Robertson, Astronomical J., 132(1) (2006), 420.

[26] R. Schild, D. Leiter \& S. Robertson , Astronomical J., 135(3) (2008), 947.

[27] Arun Pati and S.L. Baurstein, Phys. Rev. Lett., 98(8) (2007), 080502.

[28] Since here $|d r / d t|=\left|\dot{R} / R^{\prime}\right|$; partial derivatives effectively behave like total ones. Hence, the allegation by Kundt (arXiv:0905.1028) that I confused partial derivatives with total ones is incorrect. He overlooked that I was considering noncomoving $R=$ const observers. 